\title{
EL ARGUMENTO DEL LENGUAJE PRIVADO (I)
}

\author{
ENRIQUE VILLANUEVA \\ Universidad Nacional \\ Autónoma de México
}

Desde hace 21 años se discute con cierta obsesión la posibilidad o imposibilidad de un Lenguaje Privado. ${ }^{1}$ La multiplicidad de temas que engloba la denominación 'Argumento del Lenguaje Privado'* - denominación que no disputaré ha provocado reacciones extremas; unos quieren ver en el ALP un paradigua de argumento con resultados devastadores para las posiciones cartesianas en Filosofía de la Mente y Metafísica de la Persona ${ }^{2}$ mientras que otros sólo ven una serie de afirmaciones confusas que de ninguna manera llegan a constituir un argumento; la disputa se extiende aún a lo que el supuesto argumento prueba. En esta primera parte me propongo presentar una línea de la disputa; en una segunda parte examinaré los desarrollos posteriores que llegan hasta nuestros días. La cantidad de aportaciones sobre el ALP es

- De aquí en adelante usaré ALP para 'Argumento del Lenguaje Privado' y LP para 'Lenguaje Privado'.

1 La discusión sobre Lenguaje Privado la originó Wittgenstein. Casi todos los que escriben sobre este tema lo hacen a propósito de las afirmaciones de Wittgenstein. Sin embargo, esta forma de discusión acrecienta las dificultades. Por esta razón y porque el propósito de este ensayo es la historia filosófica y no la biografía filosófica he optado por usar la denominación 'Argumento del Lenguaje Privado' como un sustituto parcial de lo que Wittgenstein dijo, quizo o debió decir. [Las citas hacen referencia a los autores y las obras señaladas en la bibliografía.]

2 Varios autores han estudiado a filósofos específicos para probar esto. Así por ejemplo, Kenny [1] ha interpretado a Descartes como un sostenedor del LP. Lo mismo hace Hacker con Locke. La sospecha existe de que los filósofos Cartesianos desde Descartes hasta Russell y Husserl suponen que la idea de un LP es coherente y ocupa un lugar primario en nuestra teoría del mundo y de las personas, de ahí la importancia que algunos confieren al ALP. 
enorme $^{\mathrm{s}}$ y ello me obliga a ser selectivo tanto en temas como en autores.

\section{La interpretación de A. J. Ayer}

En 1954, en pleno fervor Wittgensteiniano - las Investigaciones filosóficas habían aparecido en 1953- A. Ayer y R. Rhees se enfrascaron en abierta disputa sobre la posibilidad de un Lenguaje Privado. En un Simposio sostenido en la Sociedad Aristotélica en Inglaterra, Ayer sostuvo que para un hombre como Robinson Crusoe era perfectamente posible tener y hablar un Lenguaje Privado. No veía Ayer dificultad en introducir el vocabulario de ese lenguaje mediante definiciones ostensivas privadas."

Ayer sostuvo que no había problema en que Crusoe inventara un lenguaje para sus sensaciones privadas - como seguramente inventó uno para la flora y la fauna de la isla - y sostuvo también que no había imposibilidad lógica en entender el lenguaje de Crusóe aun cuando sí podía haber dificultad técnica. Para Ayer la dificultad mayor consistía en verificar o checar el uso privado que haría Crusoe con los signos que introducía y esto era una dificultad para el lenguaje público también.

No es claro cuál es el sentido de la tesis de Ayer. De acuerdo a una interpretación (J. J. Thomson) lo que dice Ayer es completamente irrelevante respecto de lo que quiere probar; de acuerdo a otra (Medlin) lo que dice Ayer sí es relevante pero sólo despeja una posible confusión en el ALP. No va en contra del ALP. Según J. J. Thomson, Ayer [1] piensa que refuta la tesis de que no puede haber un lengua. je que necesariamente sólo una persona comprende probando las siguientes cosas:

a Cfr. Saunders \& Henze. La bibliografía que allí aparece sólo recoge lo publicado hasta 1967.

- La idea de la definición ostensiva privada se puede especificar como una especie de apuntar con los ojos de la mente a lo que no es compartible ni expresable v.gr. a la cualidad dolorosa que cada uno siente y puede señalar para sí pero que no puede comunicar a los demás; éste es el objeto privado. 
(1) Que hay un lenguaje que de hecho no ha sido comprendido por nadie aparte el que lo habla.

(2) Que los reportes de sensación no son ininteligibles para otros aparte del que los hace.

Pero las tesis (1) y (2) son irrelevantes para refutar aquella tesis. En cambio, según Medlin lo que Ayer piensa es que hay dos sentidos de privado, a saber:

Privado 1: Un lenguaje que usa una persona para referirse a sus experiencias privadas.

Privado 2: Sería un lenguaje en el que la expresión del que lo habla 'podría conllevar indirectamente alguna información a los demás pero no significaría para ellos lo que significa para él.

La imposibilidad de Privado 2 sería una imposibilidad lógica.

La tesis de Ayer según Medlin es la que puede haber un LP que sea Privado 1 aun cuando no sea Privado 2; mientras que algunos piensan que si un lenguaje es Privado 1 entonces será Privado 2 porque Privado 1 implica Privado 2. En suma, según Medlin, Ayer va en contra de la tesis -xcesiva - de que cualquier lenguaje que no es público tiene que ser un lenguaje necesariamente privado y por ello mismo un absurdo de lenguaje.

Contra la interpretación de J. J. Thomson se puede decir que las tesis (1) y (2) son relevantes en esta forma:

(2) Sirve para romper la implicación que va de Privado 1 a Privado 2.

(1) Constituye un contraejemplo a la interpretación según la cual el reportar una sensación no es distinto del reportar la expresión de esa sensación (ésta es la tesis que otros han expresado diciendo que entre una 
sensación y sus manifestaciones hay una relación que no es meramente contingente; la primera versión guarda un aire conductista que ésta última parece evitar). A esto Ayer replica diciendo que sí hay algo más que la expresión (Aeusserungen) ${ }^{5}$ de la sensación (o, según la otra forma, que la relación es por lo menos en parte contingente) y eso extra es lo que se busca expresar con Privado 1 .

Es importante hacer notar dos cosas:

(i) Ayer no se compromete a la doctrina de los objetos privados doctrina que muchos de los que usan el ALP creen que debe ser asumida por todo defensor del LP. Un tal defensor del ALP debe probar entonces que esta doctrina empírica de Ayer asume, de alguna manera, objetos privados.

(ii) Ayer va en contra de la doctrina del Aeusserungen si esta doctrina pretende dar cuenta de todos los casos de auto-ascripción. No toda auto-ascripción es equivalente a la expresión natural de una sensación; hay algo empírico que es Privado, y que no recoge v. gr. la interjección ¡Ay!

Hay que observar - como se verá después en el desarrollo de la polémica - que según esta interpretación de Medlin, Ayer no va en contra del ALP y antes bien su doctrina es perfectamente compatible con él.

Según Medlin, Ayer construye el argumento así: De acuerdo con el ALP si Privado 1 se reduce a Privado 2 ni siquiera el usuario de LP podrá verificar o checar su uso de $S$. Contra esto Ayer replica:

5 La tesis del Aeusserungen opone descripción a expresión. Según ella 'me duele' no describe un dolor sino que lo expresa y en estè sentido está más del lado de la conducta que del lenguaje. La tesis que algunos defensores del ALP defendieron es la de que los reportes de sensación son expresiones y no descripciones y por ello carecen de valor de verdad. 
'Mi argumento es que puesto que todo proceso de comprobación debe finalizar en algún acto de reconocimiento, ningún proceso de comprobación puede establecer algo a menos que tomemos como válidos en sí mismos algunos actos de reconocimientos ${ }^{6}$

Esto es, Ayer dice que es arbitario suponer que un enunciado Privado 2 no puede - por ser Privado 2- ser verificado o corroborado por otro enunciado Privado 2.

Dejando a un lado a quién le toca probar o refutar la implicación de Privado 1 a Privado 2, Ayer no la toca y se dedica a refutar la tesis (2) cuando lo que debe probar es que puede haber reidentificación cuando la sensación es totalmente privada, es decir, sin manifestaciones. Ayer ofrece un argumento muy pobre en favor de esto ${ }^{7}$ que no logra eliminar la extrema arbitrariedad en las putativas aplicaciones de $S$ y por ello mismo no logra mostrar que $\mathrm{S}$ es un nombre privado.

Muy importante para el desarrollo posterior de la polémica fue el diagnóstico que hizo Ayer de por qué $S$ no puede llegar a ser un nombre; pensó que había dos supuestos detrás de esa negativa a saber:

(a) 'que para que una persona pueda darle significado a un signo es necesario que otras personas lo entiendan también's

(b) 'que es lógicamente imposible entender un signo a menos que uno pueda observar el objeto que designa o por lo menos observar alguna cosa con la que el objeto está naturalmente asociado'.

Ayer fundió, así, la teoría nominativa del significado con la teoría del significado como uso (compartido); esta hete-

6 Ayer [1], p. 277.

7 Ibid., p. 56.

8 Ibid,, p. 55.

9 Ibid., p. 55. 
rodoxia fue la que descalificó sus esfuerzos a los ojos de los defensores del ALP.

Respecto de (a) Ayer respondió que aun cuando la comprensión por los demás pueda ser psicológicamente relevante no hay nada absurdo o lógicamente contradictorio en suponer que puede haber significados que nadie aparte de su posesor entiende. Esto concuerda con el ALP; lo que Ayer no dice es si es lógicamente coherente suponer significados que nadie aparte de su poseedor pueda entender. Más tarde reconocería su error al decir:

'en cualquier lenguaje que permita referirse a individuos debe haber criterios de identidad que hagan posible que diferentes usuarios del lenguaje se refieran al mismo individuo. Esto no impediría que el lenguaje tuviera sectores privados pero significaría que mi idea de que esos sectores privados pudieran absorber a los sectores públicos no era sostenible'..$^{\text {10 }}$

Por lo demás, la cita deja ver la conexión fáctica entre la defensa de un LP y el sostener un programa fenomenalista. En lo tocante a (b) Ayer atribuyó al ALP la tesis nominativa del significado causando con ello gran confusión pues de ahí en adelante se dio por sentado que la razón por la cual $S$ no puede ser un nombre es porque otros no pueden observar lo que $S$ designa y las observaciones de Crusoe mismo tampoco pueden ayudar según se vio antes. (Posteriormente veremos que Crusoe queda descalificado por la razón opuesta, a saber, por una tesis paralela a la tesis del Aeusserungen según la cual no es posible aceptar que él pueda auto-observar un dolor). Ayer pensó que ésta era una demanda excesiva que precluía, por ejemplo, la significatividad de sentencias del pasado. Si se elimina esta demanda no hay dificultad en observar indirectamente y por lo tanto en entender el lenguaje privado de este Crusoe.

10 Ibid., p. 277. 


\section{La versión de Strawson}

Strawson introdujo la noción de "criterio" ${ }^{11}$ dentro de la discusión del ALP en una forma tal que resultaba que el ALP implicaba un conductismo filosófico. Según Strawson lo que torna privado al lenguaje es el hecho de que la referencia de las palabras son sensaciones y estas son algo privado; así, el lenguaje del que él se ocupa no es necesariamente privado. Según Strawson, hay una tesis fuerte y una débil: la tesis fuerte dice que ninguna palabra nombra sensaciones; la tesis débil dice que ciertas condiciones se deben satisfacer si se han de poder ascribir sensaciones a las personas que las tienen. Ahora bien, esta tesis débil se transforma en la tesis fuerte cuando se introduce la tesis de los criterios; es en ese momento cuando el horror a lo privado torna el ALP en algo excesivo e implausible. Veamos, según Strawson la razón por la que no hay criterio de corrección para la aplicación de los nombres a las sensaciones es porque los defensores del ALP introducen escepticismo en el uso de la memoria. Al igual que Ayer, Strawson piensa que esta razón anularía a un lenguaje público también v. gr., un lenguaje de colores. Por otra parte, Strawson no ve dificultad en introducir un nombre privado: se introduce por inducción a partir de alguna característica recurrente en las sensaciones.

Strawson cree encontrar otro argumento contra el LP: si 'me duele' no puede dudarse ni es una identificación me. diante criterio entonces será equivalente a un grito (sólo que se profiere en base a educación recibida). Luego 'dolor' nó es ni el nombre ni la descripción de una sensación. Sólo hay nombre o descripción donde hay identificación o reco. nocimiento y estos sólo se dan donde hay criterio; puesto que no hay criterio en las auto-ascripciones, 'dolor' no es un nombre en esos casos; luego, no hay nombres privados. Así,

11 La noción de 'Criterio' es una de las más controvertidas. (Cfr. Kenny [3]). De acuerdo a ella ' $X$ es un criterio de $Y$ ' si $X$ es evidencia na-induc. tiva de Y. Así, por ejemplo, se dice que el quejarse, revolverse, etc. son crterios de que la persona en cuestión tiene dolor. 
según Strawson para eliminar la posibilidad de los nombres privados y con ella la de un LP, se estipula que las auto-ascripciones no son nombres sino 'expresiones' (aeusserungen) y se concluye que no hay nombres para las sensaciones. La tesis débil se ha transformado de esta manera en la tesis fuerte.

Para Strawson este exceso debe corregirse: sí hay nombres de sensaciones -este es un hecho empírico- y la condición de que los haya es que ellas se manifiestan en la conducta de las personas; reconocer la existencia de esta condición es muy diferente a decir que por ejemplo, el dolor sólo es la exclamación. Obviamente el dolor es algo más que la exclamación pero sin la exclamación no podríamos identificarlo ni nombrarlo. El dolor tiene hecesidad de criterios que residen en la conducta pero es un error suplantar el dolor por sus criterios. Nuestro lenguaje de dolor, por ejemplo, requiere la existencia de criterios pues sin ellos no habría ascripción de dolor y no habría lenguaje de dolor; y es porque hay criterios de dolor que no se pueden introducir dudas filosóficas acerca de la experiencia de dolor de otras personas ni acerca del conocimiento que tenemos de esas experiencias. Esto es de acuerdo a Strawson lo que es legítimo concluir en el ALP. Strawson advierte: quien sostenga lo contrario, esto es, quien sostenga que de todas formas un LP es posible, no cae en el sin sentido o la ininteligibilidad sino sólo en afirmaciones vacuas y sin propósito. Esto debe bastar, cualquier otra demanda es exagerada y paranoica.

Es claro que Strawson no tomó en cuenta la privacidad filosófica que afecta el LP. Probablemente pensó que ningún filósofo en su sano juicio asumiría tal tesis. Malcolm protestaría inmediatamente por esto. De todas formas, Strawson presentó una interpretación general y comprehensiva que alimentó la polémica.

La respuesta a Strawson y Ayer asumió dos formas: una esencialista y otra pragmática: los primeros en blandirlas fueron Malcolm y Rhees, respectivamente. En lo que sigue 
voy a ocuparme solamente de la respuesta 'esencialista' o fuerte que culmina en Kenny.

\section{La defensa de Malcolm}

Los defensores del ALP pensaron que ésta era una confianzuda interpretación llena de sentido común y en cierta forma grosera. Malcolm se aprestó a responderla arguyendo que en el texto de las Investigaciones había un argumento serio, profundo y concluyente en contra de la posibilidad de un LP y que este argumento quitaba al Cartesiano la posibilidad de expresar - aún para sí mismo— su teoría dualista. Malcolm recalcó que la concepción de un LP va más allá del caso de un Robinson Crusoe pues un LP no lo entiende nadie más, pero lo que es más, nadie lo podría entender. Por esta razón tanto Ayer como Strawson cometen un grave error al pensar que un LP es un lenguaje que, de hecho, nadie, aparte del poseedor, entiende. Según Malcolm la idea de un LP es una idea extrema radicalmente absurda cuya gravedad ha venido escapando a los filósofos.

Los empiristas ingleses asi como los cartesianos y todos aquellos que introducen la idea de construir o fundar el "mundo externo" o de justificar nuestras ascripciones mentales a otras mentes, los fenomenalistas como los teóricos de los datos sensibles y de la experiencia inmediata, asumen la premisa de que en mi propio caso, las ascripciones mentales son significativas y verdaderas pero resultan cuestionables en el caso de los demás; o por lo menos de que éstas nunca podrán ser tan evidentes como las auto-adscripciones. La idea es pues la de que hay una primacía en las autoads. cripciones, primacía que se manifiesta en el hecho epistemológico de que conozco, concibo, pienso o entiendo mis propios estados mentales aun cuando no entienda los de los demás. En este sentido uso un Lenguaje Privado para hablar de mis propios estados mentales lenguaje que por su naturaleza privada los demás están precluidos de entender. Esta imposibilidad se especifica de esta manera: los demás no 
pueden entender lo que significa $S$ por ejemplo, porque no pueden saber si designa algo; según esto, para ellos $S$ es un mero ruido. Así pues, el Lenguaje no es él mismo privado sino que se torna privado porque lo que le da sentido o inteligibilidad es filosóficamente privado. Este es otro de los errores de interpretación de Strawson, de acuerdo con Mál. colm. El ALP no habla de sensaciones simplemente sino de sensaciones como las concibe el Cartesiano, a saber, como objetos privados. Sólo cuando las sensaciones se conciben como objetos privados carece de sentido el nombrarlas. La estipulación Cartesiana es entonces esta: $S$ es un nombre privado en cuanto refiere a un objeto privado. De esta manera se enlazan la teoría nominativa del significado con la idea del objeto privado. La cuestión que se suscita entonces es esta: ¿en qué sentido se puede hablar de lenguaje o sentido si 'sentido' ha sido absorbido por 'objeto' en la situación privada ${ }^{12}$ Ahora bien, el juego con la idea del LP corre en esta forma: al Cartesiano por ejemplo, se le ofrece la idea de un LP como una idea que recoge lo que él considera esencial y le da forma; una vez que el Cartesiano acepta esta idea el ALP procede a mostrar que la idea de un LP es una idea incoherente. A este argumento le llamó Malcolm el argumento interno observando que tiene la forma de la Reductio ad absurdum. Según otro argumento -l argumento externoes imposible adquirir los conceptos mentales privadamente para después aplicarlos a los demás porque la idea de una tal transferencia es vacua. Los puntos sobresalientes del argumento interno de Malcolm son los siguientes:

i) Para que un signo se convierta en palabra se necesita que obedezca una regla.

ii) La regla debe ser independiente del signo.

iii) El signo puede usarse correcta o incorrectamente se-

12 Cfr. Geach, p. 4. Ginet [1] concede que pueda haber una referencia absolutamente privada pero en ese caso no habría un nombre común para ella y por lo tanto no habría lenguaje que la expresara. Rossi (Diànoia, 1963) dice que $S$ no es un flatus vocis pero tampoco llega a ser lenguaje. 
gún se siga o no la regla del mismo. Para decidir esto se puede apelar a los criterios de aplicación. ${ }^{13}$

iv) En el LP el uso de 'S' no permite distinguir entre el uso correcto y el incorrecto del mismo prueba de ello es que lo que diga el 'usuario' es lo que cuenta como correcto.

v) No es posible hacer inducciones o apelar a la memo. ria para llegar a establecer que tiene sentido hablar de un uso correcto $o$ incorrecto de ' $\mathrm{S}$ '.

iv) Todo es arbitrario en un LP.

Así, de acuerdo a Malcolm la idea misma de un LP carece de sentido pues ni siquiera se puede hablar de palabras, reglas, aplicaciones, etc.

Inmediatamente se desató una enorme polémica acerca de las 'reglas' 'criterios' 'memoria' 'sin sentido' etc. El veredicto común fue que Malcolm construía el ALP en una forma débil pues o bien suponía lo que debía probarse o se concedía todo negándole al defensor del LP aquello a que tenía derecho.

De las publicaciones de Ayer y Strawson se desprendió una interpretación de Wittgenstein de acuerdo a la cual las $I n$. vestigaciones preconizaban un conductismo tajante que negaba el hecho empírico de nuestra vida psicológica y del len. guaje que usamos para hablar de ella. La introducción de la idea de Criterio sólo embozaba y daba apariencia de refina. miento a un crudo conductismo, de origen fiscalista. Así, las Investigaciones sólo daban expresión literaria al positi. vismo de los años 30 en Filosofía de la Mente. Dos divisas abanderaron los nuevos embates, contra la interpretación de

13 Malcolm le imputa a Strawson otra confusión más a propósito de la tesis del Criterio. De acuerdo a ésta Strawson dice que el ALP confunde el criterio de v.gr. el dolor con el dolor mismo y cae así en Conductismo. Esto no es así, dice Malcolm, dolor y criterio de dolor son cosas distintas pero no habría dolor si no hubiera criterios del dolor. Eeta es la razón por la que no puede haber dolor privado.

14 Las premisas (iv) $y$ (v) suelen interpretarse como una mezcla de verificacionismo y escepticismo sobre la memoria. 
Malcolm del ALP, por un lado, el rechazo del Conductismo filosófico y por el otro, el rechazo de un argumento tan pre. tencioso como el ALP. La noción de 'experiencia' estaba le. jos de ser despreciable y argumentos como el ALP estaban lejos de ser argumentos válidos y de probar que no pudiera haber lenguajes privados. Las cosas eran menos tajantes y había lugar para muchas posiciones alternativas; no se po. día decidir el asunto con arrogancia apriorística.

La siguiente etapa de la disputa asumió asi, las siguientes características:

Por el lado Wittgensteiniano

i) Acérrima defensa del apriorismo que declara sin-sen. tido el LP.

ii) Machaqueo y repetición en los textos de Wittgenstein a fin de defender las ideas de 'regla', 'criterio', etc.

iii) Construcción y refinamiento adicional del argumento en contra del LP.

Por el lado del ataque al ALP

i) Ataque al apriorismo y al esencialismo.

ii) Ingenioso e ingenuo trabajo de buscar alternativas.

iii) Deseo de introducir las experiencias en la Filosofía de la Mente. ${ }^{15}$

iv) Intentos de argumentar en otras formas contra el dualista. $^{16}$

4. El Simposio entre Castañeda, Chappell y Thomson

H. N. Castañeda en un esforzado ensayo trató de ir a fondo en la discusión del ALP. Con similar pundonar al de los optimistas defensores del ALP que concluyen diciendo que la

15 Muy importante a este respecto es el artículo de H. P. Grice The Causal Theory of Perception' en Proceedings of the Aristotelian Society (XXXV, 1961).

${ }_{16} \mathrm{La}$ influencia del pensamiento de Quine es la más sobresaliente entre estos intentos. 
idea de un LP es un sinsentido, Castañeda sostuvo que la idea de un LP es perfectamente concebible y que el ALP es inválido. Para ello interpretó las afirmaciones de Malcolm en forma por demás directa. Castañeda pensó que si se intentaba una discusión seria de la idea de un LP debía determinarse el sentido en el que un LP es un lenguaje y el sentido en el que es privado. Como sobre esto no había claridad, Castañeda suplió la deficiencia proponiendo varios sentidos de privacidad y especificando la idea de lenguaje. Así propuso que se concibiera al LP como teniendo conectivas, cuantificadores, etc. Siguiendo a Malcolm, Castañeda pensó que la privacidad deriva de la idea de Objeto Privado, idea en la que discernió seis sentidos distintos. También distin. guió siete postulados sobre la noción de dolor. Así armado pudo presentar ejemplos de LPs en ciertos de los sentidos de objeto y de lenguaje que formuló pero no en otros.

Castañeda advirtió la posibilidad de que la doctrina de que el verbo 'sé o conozco' no se puede usar con sentido en auto ascripciones como 'me duele' pudiera usarla algún Wittgensteiniano para asentar que 'dolor' no es un objeto privado que sólo uno puede conocer y que por ello mismo la cuestión de un lenguaje para tal objeto privado ya no surge. ${ }^{17}$

Por esta vía rápida ese defensor Wittgensteiniano del ALP querría eliminar la posibilidad de que las sensaciones sean un caso de LP; de ser este el caso habría que buscar otro ejemplo. Resulta casi increíble que alguien pudiera presentar un argumento tan malo como ese en defensa del ALP. Sin embargo, hay casos.

Siguiendo a Malcolm, Castañeda piensa que una premisa crucial en una forma del ALP consiste en el cargo de que en un LP no puede haber comprobación independiente de que se está siguiendo o no la regla que rige el uso del sigo $S$. Este cargo lo interpreta Castañeda como la imposibilidad de autocorrección.

17 Castañeda [1], p. 140 y ss. Véanse más abajo las tesis de Cook y Kenny. Con esto Castañeda salió al paso de lo que muchos Wittgensteinianos afirmaban entonces. 
Castañeda siguiendo la línea de Ayer no ve dificultad en que el putativo usuario del LP se auto-corrija recurriendo para ello a otras experiencias de objetos privados, a otras palabras, a sus recuerdos, a las leyes que descubre entre esos objetos privados o en fin, a otras inducciones que puede hacer. Así, según Castañeda puede haber ambos error o equivocación sustancial en el LP y corrección de la misma. El hecho de que otros no puedan corregir no demuestra que no puede haber corrección en el LP y si se recurre al escepticismo para decir que no hay certeza en esas correcciones Castañeda (con Ayer) replica que lo mismo sucede con el lenguaje público. Por tanto, no sólo muestra Castañeda que la premisa crucial del ALP carece de evidencia o es implausible sino que la quiere probar falsa y con ello quiere demostrar que el ALP es inválido y que existen LPs. Esto en verdad es contestarles a los defensores del ALP en su propio terreno.

Los Co-simposiastas de Castañeda, J. F. Thomson y V. Chappell expresaron dudas acerca de la existencia de un ALP. Thomson dijo no entender la razón para afirmar la premisa - sumamente fuerte - de que nadie aparte del posesor de las sensaciones puede entender el lenguaje que habla de ellas; debido a esto y a lo que consideró una pobre reconstrucción por Castañeda y otros del putativo ALP, Thompson expresó escepticismo en la existencia del ALP. Chappell fue más radical; junto con Thomson acusó a Castañeda de cometer una Ignoratio Elenchi al ignorar el tipo de lenguaje que Malcolm y otros discuten, a saber, un lenguaje que nadie aparte de su putativo posesor puede entender. Según Chappell, Castañeda da por sentado que ese lenguaje existe y pasa a construirlo y por ello se equivoca tan rotundamente pues lo que se discute es si dada la idea filosófica de la privacidad podria haber un lenguaje; en el ALP lo que se maneja es una idea vaga de lenguaje y el propósito del argumento es mostrar la vacuidad de la idea.

Exista o no un ALP, Chappell y Thomson objetan a Cas- 
tañeda que el LP que él discute no es el que Malcolm y otros atacan. A esto replicó Castañeda con la irrelevante afirmación de que Malcolm no tiene derecho a estipular qué sea un LP. Chappell, sin embargo, no se contentó con querer mostrarle a Castañeda su ignoratio sino que quiso probar que si los términos del LP son privados de nada sirve al putativo usuario del LP recurrir a los elementos 'públicos' o privados que Castañeda menciona pues ellos no le permitirán distinguir entre usos correctos o incorrectos y por lo tanto no le sirven para probar que el dicho usuario pueda cometer erro. res al usar esas palabras privadas.

Para mostrar esto Chappell construyó un argumento cuya premisa crucial reza así:

"en el caso del usuario de un signo o lenguaje privado no puede haber apelación a algo externo a la mente del sujeto... a algo "no subjetivo" "18

y por tal razón el signo $S$ no es un signo y el uso de $S$ no es una actividad linguística. Chappell se dedica luego a mostrar que uno por uno de los candidatos que Castañeda propone para probar que sí se puede introducir la distinción correcto-incorrecto fallan.

Sólo voy a examinar el argumento contra uno de los candidatos, a saber, el de que el putativo usuario del LP puede recurrir a otros objetos privados para decidir si ha aplicado $S$ correctamente o no. ¿Cómo pueden esos objetos privados decir esto? ¿Cuáles objetos privados se usarían para ello? Sólo objetos privados presentes o pretéritos. Pero ¿en qué sentido esos objetos pueden decidir que $S$ se está usando correctamente? Acaso es ¿porque hay un escrutinio o examen más ceñido del 'uso' de $S$ ? Aún concediendo que esto sea inteligible Chappell responde:

“Pero eso sólo atiende a mi convicción; la cuestión es la 18 Chappell, p. 162. 
de si estoy en lo correcto, esto es, la de si esta es la pala. bra que usé para objetos de este tipo en el pasado."10

Eliminando así el recurso a otros objetos privados queda eliminado el uso de recuerdos, impresiones, el de otras pa. labras y el de las generalizaciones inducidas a partir de esos objetos privados. Por último, el recurso a 'conexiones lógicas' entre $S$ y otros términos del lenguaje público haría que $S$ perdiera su carácter privado y por ello también debe ser re. chazado. En esta forma Chappell concluye que Castañeda fracasa en su intento de mostrar que puede haber lenguajes que sean privados filosóficamente.

En su contraréplica Castañeda respondió - siguiendo en esto a Ayer- que, contra Chappell, las razones que sirven para eliminar el recurso a otros objetos públicos o privados sirven para probar que tampoco en el lenguaje público puede aplicarse la distinción correcto-incorrecto. Castañeda concluyó afirmando creer que había refutado el ALP aun cuando concediendo que puede haber cierto tipo de LPs. Esta conclusión por Castañeda dejó ver que se había llegado a un impasse (especialmente con Chappell). Más tarde volvere. mos sobre algunos de los tópicos tratados aquí.

La discusión del ALP en este simposio dejó asentada" una forma de ver el ALP forma que ha proseguido hasta A. Kenny y cuyo desarrollo voy a perseguir en las páginas que siguen.

\section{Las aclaraciones de J. W. Cook}

J. W. Cook intervino en la disputa para aclarar la razón que explica ambos, la fuerte confusión de Castañeda y la perplejidad de Thomson. Esa razón yace en una concepción de la privacidad; el propósito de Cook fue exponer esa con. cepción y mostrar su carácter absurdo. Para ello formuló el siguiente argumento:

19 Chappell, p. 163. 
$P_{1}$ Nadie puede sentir (experimentar, tener contacto in mediato con) las sensaciones de otra persona.

$\mathrm{P}_{2} \mathrm{La}$ vía apropiada y necesaria para llegar a conocer la sensación que siente otra persona es la de sentir la sensación de esa persona.

, $\mathrm{P}_{3}$ Quienquiera que tenga una sensación sabe que la tiene porque la siente y cualquier cosa que se puede saber que existe porque se la siente no se puede conocer (en el mismo sentido de 'conocer') que exista de ninguna otra forma.

C Nadie puede saber qué sensaciones tiene otra persona. ${ }^{20}$

Según Cook de la conclusión $\mathrm{C}$ se sigue que no se pueden enseñar los nombres de sensaciones a otras personas; presumiblemente también se sigue que ningún otro puede entender esos nombres porque para entenderlos se necesitaría conocer aquello a que se refieren y por lo tanto que el lenguaje de sensaciones es un LP. La idea de Cook es mostrar que esta concepción del conocimiento de sensaciones es ininteligible y no sỏlo elimina el caso de 'Los otros' como pretende el Cartesiano sino que resulta fatal para el caso propio (para el uso solipsístico) y al hacerlo ver así quiere eliminar el motivo de la idea del LP, a saber, que hay objetos privados para los cuales puede haber un lenguaje. Una vez más es la idea del objeto privado la que ocupa el centro de la discusión. Cook piensa que hay dos errores en la interpretación de las sensaciones, a saber:

(i) Las sensaciones son privadas y por ello no hay nombres de sensaciones, en realidad, aunque aparentemente los haya.

(ii) Las sensaciones son privadas pero tienen nombres, luego, el lenguaje de sensaciones que tenemos es un ejemplo de Lenguaje Privado.

20 Cook, p. 289-96. 
La primera (i) es la interpretación de Strawson y Pitcher, la segunda (ii) es la de Castafieda. (i) es el precio que se paga por eliminar el LP, (ii) es el precio de reducir las sensaciones a objetos privados. Empero, según Cook ambas (i) y (ii) están completamente equivocadas y esto es lo que muestra el argumento arriba expuesto. Veamos:

De acuerdo a un Cartesiano $P_{1}$ expresa una imposibili. dad lógica' pero de acuerdo a Cook no expresa nada. $\mathbf{P}_{1}$ parece decirnos algo sólo porque parece ser de la misma forma lógica que 'nadie puede tener la túnica de César' pero esto no es así pues mientras uno puede identificar la túnica y luego preguntar de quién es, uno no puede hacer lo mismo con 'dolor'.

$P_{2}$ y $P_{3}$ expresan una condición necesaria según la cual para conocer qué sensación tiene una persona uno tiene que sentir la sensación y a menos que uno la sienta, no la conocerá. Según Cook no hay tal condición porque para que 'conozco o sé' sean expresiones de certeza en enunciados en primera persona tiempo presente es necesario que tenga sentido que el que habla se pueda equivocar y esto nunca puede suceder con 'me duele'. Cook piensa que la única prueba en favor de esto - de la incorrigibilidad- que se puede ofrecer es o bien ejemplificar esa verdad con usos del lenguaje o hacer ver que los únicos errores que pueden cometerse son errores al describir un dolor o una sensación y no errores acerca del hecho de que duele. Por lo tanto, $\mathrm{P}_{2}$ y $\mathrm{P}_{3}$ carecen de sentido al igual que $P_{1}$ y por ello lo que parecía un argumento se desvanece como una alucinación sin dejar más que la huella de palabras cuyo sentido existe sólo en otras áreas o juegos del lenguaje. ${ }^{22}$

Ahora bien, ¿por qué decir que las premisas del argumento A "carecen de sentido" y no limitarse meramente a decir que son falsas? Este es quizá el aspecto más interesante de Cook quien parece creer que las observaciones de Wittgen-

21 Ibid., p. 302.

22 Ibid., p. 293-4 y 295. 
stein en las que Cook apoya sus argumentos descansan en una fuerte teoría, una especie de super semántica filosófica. Cook interpreta lo de los juegos del lenguaje como Categorías Lingüísticas que demarcan clases o tipos de palabras y sentencias, de suerte que cuando se mezclan o confunden esos tipos el resultado es el sinsentido o la ininteligibilidad. Así dice que 'el abrigo de mi vecino' no es como 'el dolor de mi vecino' pero algunos ceden a la analogía gramatical y caen en el sinsentido. Esta es una interpretación fuerte que a muchos ha molestado - como tendremos oportunidad de señalar después - pues han creído ver en esas afirmaciones una forma radical de esencialismo. Sin embargo, Cook nunca basa sus declaraciones de sin-sentido en la existencia de reglas o criterios como lo hacen otros y es esto quizá lo que torna su posición aún menos digerible a los ojos de muchos.

\section{La disputa sobre las reglas privadas}

En el artículo sobre LP en la Enciclopedia de la Filosofía de Edwards, ${ }^{23}$ Castañeda atacó también la idea de que uno no puede obedecer una regla privada. Castañeda objetó que no es una condición de una regla el que exista la posibilidad de desobedecerla; hay reglas que al pensar que uno las obedece ya las está obedeciendo.

En forma similar arguye J. J. Thomson, ${ }^{24}$ quien dice que en ejemplos como:

(1) "Mamita escribe: 'Siempre que te sientas decaída, piensa en tu mamita'. (Ello te levantará el ánimo)."

(2) "Si tienes dolor, piensa que tienes dolor" entender la regla implica obedecerla.

A esto contestó C. Ginet diciendo que ambos Castañeda y Thomson resbalaron ante un caso muy común de desobediencia de reglas, a saber:

23 Castañeda [2], p. 461-2.

24 J. J. Thomson, p. 191. 
'aún en el caso de reglas muy fáciles de entender es po. sible que alguien piense que entiende la regla cuando no es asi. ${ }^{25}$

Así en el primer ejemplo citado, contrario a los propósitos de la madre, la hija puede tener una idea demasiado magra de su madre o de la institución materna en general y al malentender en esa forma el espíritu del consejo materno, aun cuando piensa que obedece al consejo, llegar a resultados aún peores que los de la situación inicial.

De esta forma Ginet salva la premisa del ALP de acuerdo a la cual toda regla debe ser desobedecible premisa que se especifica negativamente diciendo que 'Pensar que uno obedece una regla no es obedecer la regla'.

\section{Argumento del Lenguaje Privado y Verificacionismo}

J. J. Thomson recogió la sospecha formulada por muchos de que la premisa clave del AEP descansa en verificacionismo. J. J. Thomson se pregunta ¿Por qué no es posible que $S$ sea un nombre en un LP? ¿Por qué no puede checar sus propias aplicaciones el usuario del LP? ¿Por qué, en suma, no puede seguir una regla privada? Bueno, responde ellà, porque el usuario del LP no puede encontrar, descubrir o llegar a darse cuenta de que su ocurrencia mental no es $S$ o $E$ o cualquier otro nombre que él quiera darle. Por ahí llega a concluir

'To que se requiere es un encontrar fuerte o conclusivo - sea porque las buenas razones no bastan o porque donde no puede haber encontrar fuerte tampoco puede haber buenas razones'. ${ }^{28}$

Según esto si Malcolm y otros declaran que no tiene sentido hablar de nombres comunes en un LP lo dicen en base

25 C. Ginet [2], p. 363.

26 J. J. Thomson, p. 198. 
a una teoría verificacionista del significado; pero además postulan una segunda cosa, dicen que el único tipo de ascripciones que es posible verificar son aquellas que refieren a algo físico, a la conducta. De esta manera se deshacen del fenomenalismo y abrazan la tesis fisicalista.

Según J. J. Thomson todo el estruendo causado por Malcolm y sus seguidores se reduce a un cambio en las palabras, cambio por demás superficial pero engañoso. Sin embargo, cuando uno lo mira con cuidado se reduce a algo sim. ple, crudo y común, a saber, a la tesis verificacionista del significado de los años $30 \mathrm{~s}$. J. J. Thomson concluye con dos puntos finales; primero, nos recuerda que el tratamiento del significado en términos de verificación envuelve circularidad y, segundo, que Malcolm y los otros prestan un pobre servicio a Wittgenstein al interpretarlo como ofreciendo argumentos en favor de tesis filosóficas en contra de su ad. vertencia expresa.

Creo que es posible argüir en contra de la circularidad que J. J.'Thomson cree encontrar en su ALP. De otra parte, es interesante notar que se tome la teoría verificacionista no en su sentido original y propio, a saber, como delimitando la ciencia de la Metafísica sino en este sentido en que J. J. Thomson la toma como dando las condiciones necesarias y suficientes de toda significatividad. Es impensable que alguien pueda pretender hacer uso de una tesis tan rara y ciega. Ad hominen uno puede argüir que si el ALP es como J. J. Thomson pretende que es, resulta un ejercicio tanto pueril como inútil el dedicarse a refutarlo; esto sería literal. mente como dispararle a un enemigo muerto. Sin embargo, más allá de J. J. Thomson la sospecha persiste de que la premisa crucial del ALP descansa en una negativa por parte de Malcolm y sus seguidores a permitir nombres privados negativa que se traduce en regalarse todo el juego y eliminar al adversario antes de disputar con él. La sospecha de verificacionismo debe ser eliminada para que sea posible tomar seriamente al ALP. 
A restablecer el ALP y despejar varios tipos de sospechas se ha avocado A. Kenny en sucesivos artículos. Me interesa comentar sobre el punto señalado más arriba, a saber, ¿porqué no puede haber Nombres Privados y por tanto LP?

\section{El ALP y las condiciones de la proposicionalidad}

A. Kenny también piensa como Malcolm que la idea de un LP está implicada en la Epistemología y la Filosofía de la Mente de los que asumen experiencias privadas. El ALP parte de esta implicación y deduce que en un LP nada se puede decir ni preguntar. El ALP descubre dos errores fundamentales: el error de concebir las experiencias como privadas y el error de pensar que las palabras son parte del lenguaje porque tienen significados adquiridos por ostensión privada.

Veamos primero el error acerca del lenguaje. La versión según la cual $S$ refiere a la sensación de dolor por ostensión privada es una simplificación; para que pueda haber 'referencia a' se necesita una base compleja de costumbres, circunstancias, etc. No basta la mera experiencia como tampoco basta la mera conducta. Si se enfatiza la conexión de v. gr. 'dolor' con los criterios o conducta de dolor es para mostrar la insuficiencia de las experiencias no para hacer de los criterios algo suficiente. Este es uno de los errores de la interpretación de Strawson según vimos anteriormente.

La tesis de la privacidad filosófica pretende derivarse del "hecho" de la incomunicabilidad o del "hecho" de la inalienabilidad. La incomunicabilidad es epistemológica ("No puedo conocer tus dolores') la inalienabilidad es de posesión ('Nadie puede tener mi dolor') pero también puede deri. varse de ambos hechos ('No puedo conocer tu dolor porque no lo tengo'). Kenny elimina las tres posibilidades califi. cándolas frecuentemente de 'sinsentido'; la privacidad filosófica no puede introducirse pues, a partir de ninguno de esos "hechos". La única privacidad que existe es la del se- 
creto ('Ocultaré el dolor que siento durante toda mi vida') pero ésta no crea problema filosófico alguno.

Esto muestra de acuerdo á Kenny que nuestro 'dolor' no es filosóficamente privado y por lo tanto que nuestro lenguaje de sensaciones no es un LP. Ello deja al descubierto los errores de interpretación de Strawson, Pitcher y Casta. ñeda. Pero ¿no podría haber un "dolor" similar a nuestro 'dolor' sólo que con la característica de ser incomunicable?; sería un pseudo-dolor. Luego ¿no podría yo definir $S$ de manera que refierese a ese "dolor" mediante una asociación entre $S$ y "dolor"?

Tanto Kenny como Malcolm rechazan esta posibilidad pero sin asumir escepticismo de la memoria o verificacionismo. Según Kenny sólo hay tres respuestas a la pregunta. '¿Qué quieres decir por $S$ ? y son las siguientes:

(1) Por $S$ quiero decir esto.

(2) Por $S$ quiero decir la impresión ' $S$ ' que recuerdo.

(3) Por $S$ quiero decir $\mathrm{X}$ correlato físico.

(1) queda eliminada porque

“ "esto es $S$ " no es una proposición genuina capaz de ser verdadera o falsa pues lo que le da su contenido es la misma cosa que le da su verdad: el significado del predicado se presume fijado por la referencia del sujeto' ${ }^{27}$

(2) queda eliminada también porque

'debe evocar el ejemplar-recuerdo de $S$ y compararlo con su sensación presente para ver si las dos son semejantes. Pero desde luego debe invocar el recuerdo correcto. Ahora bien, ¿es posible que a su evocación pudiera ocurrir un recuerdo equivocado? Si no puede, entonces ' $S$ ' significa cualquier recuerdo que se

27 Kenny [3], p. 219. 
le ocurra en conexión con ' $S$ ', y nuevamente, cualquier cosa que parezca correcta es correcta". ${ }^{28}$

En ambas (1) y (2) no se logra eliminar la arbitrariedad que torna vacuo el declarar a $S$ un nombre. (3) parece remediar esto pero a un costo imposible pues si aplico $S$ cuando por ejemplo mi presión sanguínea sube tendré ciertamente un nombre pero será un nombre 'público'.

Contra J. J. Thomson dice Kenny que no es verificacionismo lo que supone el rechazo de las tres respuestas sino la teoría de la bi-polaridad de la proposición definida en el Tractatus. No es que no se acepten las verificaciones privadas y se exija algo más fuerte sino que la posibilidad misma de verificar es la que no se puede introducir porque el es. pacio para la verdad o la falsedad ha sido cancelado.

Esa teoría de la proposición va en contra de la idea de la experiencia inmediata y por lo tanto, va en contra de la noción de objeto privado en la medida en que éste hace imposible la ascripción de verdad o falsedad. Así, de acuerdo a Kenny el ALP resulta de una aplicación de la tesis del Tractatus al campo de la filosofía psicológica.

Kenny apunta otra conxeión ahora entre la teoría de la proposición y las doctrinas según la cual 'me duele' por ejemplo, no es una descripción (de la cual se deriva la doctrina de que es una expresión o aeusserungen) y 'sé que me duele no es una afirmación epistemológica. Cuando se dice que 'me duele' no es una descripción lo que se quiere negar es que uno lee esa sentencia a partir de una observación de los hechos y lo que se quiere enfatizar es que a diferencia de una descripción de mi automóvil, por ejemplo, 'me duele' es un criterio de mi dolor, es parte de mi conducta de dolor y por lo tanto, parte de 'dolor' $;^{28}$ la descripción de mi automóvil, en cambio, es independiente de mi automóvil y por ello puedo decir por ejemplo, que es verdadera o falsa. Este carác-

29 lbid., p. 224.

28 lbid. 
ter no-independiente de 'me duele' - que lo descalifica como una descripción - guarda una semejanza con el carácter noarticulado de 'esto es $S$ ' —que la descalifica de ser una proposición.

Algo similar ocurre también con 'sé que me duele'..$^{\text {o }}$ Para que 'sé' o 'conozco' tenga un uso epistemológico debe poder ser una descripción verdadera de un hecho y para ello debe ser posible que el que la prefiera se equivoque pero esto no puede suceder. De nuevo, como en 'esto es $S$ ' no hay independencia y por lo tanto la adición de 'se' o 'conozco' a 'me duele' resulta vacua como una adición epistemológica.

Así pues la tesis general parece decir que cuando se cancela el espacio lógico entre un nombre y su referencia o entre una descripción y el hecho o en fin entre una afirmación de conocer y el objeto de conocimiento, se cancelan por ello mismo 'nombre', 'descripción' y 'conocimiento'. Esta es la maniobra que se hace con teorías como la del Nombre Propio Lógico y la tesis de la Experiencia Inmediata que dan sustento a la idea de un LP.

En esta forma remata Kenny una línea de argumento iniciada por Ayer. Según ella tanto opositores como defensores del ALP demandan la formulación de argumentos apoyados en tesis filosóficas. Del lado de los defensores se introducen tesis acerca de la significación, criterio, etc., del lado opuesto tesis acerca del lenguaje, las experiencias, etc. Kenny hace uso de todos esos conceptos y en forma novedosa provee respuesta a las objeciones contra este tipo de argumentos. En la segunda parte de este ensayo me ocuparé del desarrollo posterior a Kenny de la disputa sobre el ALP. 


\section{BIBLIOGRAFIA}

A. J. Ayer [1] 'Can There Be a Private Lenguage?' en Jones, ed. [2] 'Privacy' en The Concept of a Person MacMillan, London, 1963. Hay traducción castellana.

H. N. Castañeda [1] 'The Private Language Argument' en Jones, ed. pp. 132-182.

[2] 'Private Language Problem' en The Encyclopedia of Philosophy. New York, 1967.

V. C. Chappell Comentarios a Castañeda [1] en Jones, ed. pp. 155.68.

J. Cook 'Wittgenstein on Privacy' en Pitcher [1] pp. 286-323.

A. Donagan 'Wittgenstein on Sensation' en Pitcher [1] pp. 324-351.

P. Geach Mental Acts Routledge \& Kegan Paul, 1964.

C. Ginet [1] 'How words Mean Kinds of Sensations' Philosophical Review, 1968.

[2] "Wittgenstein's argument that One Cannot Obey a Rule Privately" Noûs 1970.

P. Hacker Insight and Illusion Oxford 1973.

O. R. Jones ed. The Private Language Argument MacMillan 1971.

A. Kenny [1] 'Cartesian Privacy' en Pitcher [1] pp. 352-70.

[2] 'The Verification Principle and the Private Language Argument' en Jones, ed. pp. 204-28.

[3] 'Criterion' en Encyclopedia of Philosophy, N. Y., 1967.

D. Locke Myself and Others Oxford 1968.

N. Malcolm 'Wittgenstein's Philosophical Investigations' en Jones, ed. pp. 3349.

C. E. Marks Private Language Tesis Doctoral depositada en la Universidad de Cornell.

B. Medlin 'Critical Notice of Ayer's Concept of a Person and Other Essays'. Australasian Journal of Philosophy, 1964.

D. F. Pears Wittgenstein Fontana 1971 Cap. 8. Trad. Castellana en Grijalbo, 1973.

G. Pitcher [1] ed. Wittgenstein: The Philosophical Investigations MacMillan 1968.

[2] The Philosophy of Wittgenstein Prentice-Hall, 1964.

R. Rorty [I] 'Wittgenstein, Privilaged Access, and Incommunicability'. Am. Phil. Q., 1970. 
[2] 'Verification and Transcendental Arguments'. Noûs, 1971.

[3] 'Criteria and Necessity'. Noûs, 1973.

R. Rhees 'Can There Be a Private Language?' en Jones, ed.

Saunders \& Henze The Private Language Problem. Random House, 1967.

P. F. Strawson [1] 'Critical Notice: Philosophical Investigations', parcialmente en Jones ed. pp. 27-32.

D. M. Taylor 'The Incommunicability of Content' Mind. Oct. 1966.

J. F. Thomson Comentarios a Castañeda [1] en Jones ed. pp. 168-73.

J. F. Thomson 'Private Languages' en jones ed. pp. 183-204.

Wittgenstein [1] "Notes for Lectures on "Private Experience", and "Sense Data", en Jones ed., pp. 232-275.

[2] Philosophical Investigations, Oxford, 1953.

[3] Zettel, Oxford, 1967. 
SUMMART

This is a survey paper divided into two parts; in this issue of Critica appears part I; it deals with what I shall call the strong line of the PLA.* In a second part - forthcoming - I shall develop further the strong line till nowadays and I shall also present the weak line.

Discussing the nature, scope and results of the PLA is very ambitious. Some philosophers see the PLA as a devastating argument against Cartesian Metaphysics and its Philosophy of Mind whereas others see only superficial linguistic platitudes in the PLA which have nothing to do with real philosophical problems. I shall review what both parties have to say and thus provide an introduction to this much debated topic.

\section{A. J. Ayer's Interpretation}

Ayer sees no difficulty in a Robinson Crusoe's acquisition and use of a PL. He could get one such language by private ostensive definitions. According to Ayer if there is a difficulty for Robinson's private tests there will also be a difficulty for public tests.

Ayer thinks he can refute the thesis which holds that there cannot be a PL. According to J. J. Thomson, Ayer misses the target for he goes on proving two quite irrelevant thesis, namely:

(1) That there is, in fact a language not understood by anyone besides the speaker.

(2) That sensation reports can be understood by someone else besides the speaker.

But according to Medlin, Ayer thinks that Private is a twofold concept.

Private $_{1}$ : a language a person uses to refer to his private experiences.

Private $_{2}$ : a language that 'might indirectly convey some information to others without meaning to them exactly what it means to him'.

On Medlin's view what Ayer tries to avoid is the implication from Private $_{1}$ to Private $_{2}$. If there is no implication from Private ${ }_{1}$ to Private $_{2}$, then it will be possible to build a PL and there will be something else beside the expression of e.g. pain.

* I shall be using PLA 'Private Language Argument' and PL for 'Private Language'. 
This much could be accepted by a defender of the PLA but Ayer does not leave things in that state; instead of attacking the implication from Private ${ }_{1}$ to Private $_{2}$ he sets forth to attack thesis (2) and confuses the whole issue.

Ayer also offered a diagnosis of the reasons why $S$ cannot be a name. This diagnosis has pervaded a great deal of further discussion regarding the PLA. It consists in the two following conditions: $S$ cannot be a name unless

(i) It is understood by other people.

(ii) The user could observe the object $S$ designates.

He later came to accept condition (i) and thus gave up his belief in a PL. But his diagnosis brought up many problems.

\section{Strawson's version}

Strawson introduced the notion of Criterion in his discussion. According to him, what makes a PL private is the empirical fact that such a language refers to sensations and that sensations are private. Strawson also distinguishes between a strong and a weak thesis. The strong thesis says that no word names sensations; the weak thesis says that words name sensations only under certain conditions. The weak thesis becomes the strong one in this manner: names require identification and identification requires criteria, but self-ascription is criterionless (aeusserungen) thus there are no names for sensations. Strawson considered this an excess and recomended a more lenient thesis about criteria.

\section{Malcolm's defense}

Malcolm felt that Strawson's interpretation was misleading and mistaken. In the first place Strawson did not understand that the PLA is a philosophical argument and that the PL has to be a language necessarily private. Sensation language is not a PL; it becomes private when sensations are built, as private objects. Malcolm distinguished between the two arguments: the internal one based on the notion of a rule having the form of a Reductio and the external one based on the idea of transferring one's private mental concepts. Malcolm questions the intelligibility of such a transference and thus eliminates the external argument. According to the internal argument, the idea of a rule that couldn't be neither correct nor incorrect is the unintelligible one and, if the notion of rule is not intelligible, the idea of private names or words won't be either.

Regarding the criteria, Malcolm thought that Strawson read that 
notion in a strong behaviouristic way and thus was led to his mistaken views on sensations.

\section{The Symposium between Castañeda, Chappell and Thomson}

Castañeda made a strong effort trying to clarify the sense of a PL, by distinguishing between various senses of Language and Privacy. Castañeda contested the main premiss in Malcolm's argument proposing a number of candidates that could serve the purpose of determining that $S$ was applied correctly or incorrectly; these candidates were other experiences of private objects, other words, memories, law like relationships among these private objects or inductions made from these objects. Castañeda saw no special difficulty in proposing examples of PL.

J. F. Thomson and V. Chappel co-symposiasts complained that the existence of an argument concerning Pls wasn't clear at all. Thomson said he couldn't understand the reasons behind the very strong premiss 'nobody besides its prossessor could understand the language of sensations'. Chappell said that Castañeda did not pay attention to the type of language under discussion, namely, a lan. guage that nobody apart from his possessor could understand. Thus Castañeda committed an Ignoratio Elenchi. Besides this, Chappell objected to the above mentioned candidates use of $S$ being correct or incorrect, since they were useless in deciding whether any use of these candidates would deprive the PL of its privacy. Further on Chappell set forth to show that even granting the use of the candidates they would provide no help at all because, for example, a recourse to other private objects would only touch one's own conviction, not the correctness of $S$ 's application.

\section{J. W. Cook's clarifications}

Cook tried to satisfy Thompson's misgivings and to dispell Castañeda's confusions. In order to achieve this, he formulated an argument whose main premise stated that a necessary condition for knowing a sensation is to feel the sensation itself. This premise was the source of the philosophical privacy and so the source of the idea of a PL. If one accepts this premise the conclusion will follow that no sensation words could be taught and thus that the language of sensations is a PL. Then, Cook goes on to show the senselessness of that premise - not is falsity-, For this he uses contrasts of the ordinary language that bring out the oddness of sentences like "nobody 
could have my pains'. A sort of supersemantics seems to lie behind Cook's charges of senselessness.

\section{The dispute on Private Rules}

Castañeda and J. J. Thomson objected to Malcolm's contention that there couldn't be private rules because these couldn't be disobeyed; according to them there are such rules which only thinking of obeying them is to obey them, as, for example:

'If you have pain, think that you have pain'. In these cases understanding the rule implies obeying it.

C. Ginet answered this objection noticing that "even in the case of the most easily understood sort of rule it is possible for someone to think he understands the rule when he does not." Thus in no case thinking that one obeys a rule is to obey it.

\section{PLA and Verificationism}

Why -asked J. J. Thomson- cannot $S$ be a name? Why is it impossible to follow a rule privately? All the discussion about impossibilities and senselessness is related to two philosophical theses, namely, Verificationism and Physicalism. According to the first, no sentence has meaning unless it is verifiable; according to the second, nothing counts as providing verification but what is physical. It is because PLA's defenders hold these thesis that private naming, private rules and private checks appear as unintelligible to them. But as Verificationism is in discredit because of the charge of circularity, there is no acceptable reason to reject PLs.

\section{ALP and conditions for Proposisionhood}

A. Kenny tried to overcome the main obstacle of the PLA, that is to show conclusively why 'This is $S$ ' is not intelligible when philosophical privacy is the case. According to him, the theory of proposition laid down in the Tractatus is being used to reject This is $S$ as a proposition; no verificationism is involved in such rejection.

Kenny distinguishes three 'facts' which would serve to introduce philosophical privacy, namely, incommunicability which is epistemological ('I cannot know your pains') inalienability which is by ownership ('Nobody could have my pains') and a mixture of these two ('I cannot know your pains because I cannot have them'). All three are dismissed and thus no philosophical privacy could be introduced. There is only one 'fact' which can introduce privacy, 
namely, secrecy but this one doesn't constitute a philosophical prob. lem. So, 'pain' is not philosophically private and Kenny provides an explanation of Strawson's and Castañeda's misinterpretations.

Kenny also rejects the idea of private names. His answer is a further elaboration of Malcolm's and Chappell's responses. Contra J. J. Thompson and others he claims that neither verificationism nor scepticism on Memory is required in order to reject 'This is $S$ ' as a proposition. 'This is $S$ ' is not a proposition because the three possible answers to the question 'what do you mean by $S$ ' fail. If it is answered 'By $S$ I mean this' that won't be a proposition because what gives it its content is what gives it its truth. If it is answered 'By $S$ I mean the impression ' $S$ ' that I remember' it would be necessary to have a memory-copy that would have to be compared to the present impression and two possibilities appear: they could fail to agree or not: if they cannot agree, memory is of no help because anything could do and if they can agree memory is of no help either because something else would be needed. The third answer is this: 'By S I mean X physical correlate'; this will do but there won'I be any privacy left.

The failure of 'this is $S$ ' as a Proposition has something in common with the failure of 'I have a pain' as a description and with the failure of 'I know I have a pain' as an epistemological assertion. All these failures stem from the theory of immediate experience or acquaintance which lies deep at the source of the idea of a PL.

Summary by Enrique Villanueva 